\title{
Relativistic meson spectra on ion-trap quantum simulators
}

\author{
Johannes Knaute $\mathbb{( 1}^{1,2, *}$ and Philipp Hauke ${ }^{3, \dagger}$ \\ ${ }^{1}$ Max Planck Institute for Gravitational Physics (Albert Einstein Institute), 14476 Potsdam-Golm, Germany \\ ${ }^{2}$ Department of Physics, Freie Universität Berlin, 14195 Berlin, Germany \\ ${ }^{3}$ INO-CNR BEC Center and Physics Department of Trento University, Via Sommarive 14, I-38123 Povo (Trento), Italy
}

(Received 1 August 2021; accepted 9 February 2022; published 22 February 2022)

\begin{abstract}
The recent rapid experimental advancement in the engineering of quantum many-body systems opens the avenue to controlled studies of fundamental physics problems via digital or analog quantum simulations. Here, we systematically analyze the capability of analog ion traps to explore relativistic meson spectra on current devices. We focus on the $E_{8}$ quantum field theory regime, which arises due to longitudinal perturbations at the critical point of the transverse-field Ising model. As we show through exact numerics, for sufficiently strong long-range suppression in experimentally accessible spin chain models, absorption spectroscopy allows for the identification of the low-lying meson excitations with a good degree of accuracy even for small system sizes. Our proposal thus opens a way for probing salient features of quantum many-body systems reminiscent of meson properties in high-energy physics.
\end{abstract}

DOI: 10.1103/PhysRevA.105.022616

\section{INTRODUCTION}

Emergent phenomena of quantum many-body (QMB) systems play a major role in condensed matter and particle physics [1-3]. The recent progress of quantum simulation technologies [4-6] in controllable platforms such as ion traps [7-10] has opened the prospect of treating fundamental effects and systems beyond the capability of classical computers. Various trapped-ion experiments have already unveiled static and dynamical properties of quantum matter [11-23] as well as lattice gauge theories [24,25].

In this work, we are interested in using trapped-ion devices to study mesons, which are nonperturbative bound states consisting of two subparticles or charges. They appear prominently in quantum chromodynamics (QCD), the theory of strong interactions within the standard model of particle physics, where a quark-antiquark pair is confined by a flux tube. Their properties and phenomenology is of key importance for the understanding of heavy-ion collisions, which provide an experimental way of studying far-from equilibrium dynamics relevant to the physics of the early universe [26]. Beyond particle physics, mesons exist also in condensed matter systems, in particular Ising spin chain models, where symmetry breaking longitudinal fields [27] or long-range interactions $[28,29]$ can confine domain walls into mesons. The

\footnotetext{
*johannes.knaute@aei.mpg.de

†philipp.hauke@unitn.it
}

Published by the American Physical Society under the terms of the Creative Commons Attribution 4.0 International license. Further distribution of this work must maintain attribution to the author(s) and the published article's title, journal citation, and DOI. Open access publication funded by the Max Planck Society. existence of mesons in the spectrum has severe consequences for both static and dynamical properties of the QMB system at zero and finite temperature. Some of the diverse implications for entanglement, correlations, and thermalization are theoretically studied in [30-39]. In long-range models, the existence or absence of meson states has also profound implications on the emergence of anomalous cusps in dynamical quantum phase transitions [40-44].

Analog quantum simulations can implement such spin Hamiltonians and therefore provide access to meson features. Experimental evidence of dynamically induced magnetic domain wall confinement was first provided in [45] and [46] by analyzing the meson impact on correlation and entanglement spreading after quantum quenches. Recently, the authors of [47] proposed a protocol to measure fluctuations and dissipations in quantum simulators (see also [48]), and illustrated a way of obtaining spectral information of the meson system from their relations. An improved error mitigation technique for the extraction of meson masses on quantum simulators was discussed in [49]. While these systems are currently most developed in $(1+1)$-dimensional simulations, their phenomenology can provide important insights that are relevant across dimensions. For example, the recent papers [50-52] explored the capabilities of quantum simulations for real-time string breaking and meson scattering [53]. All these studies focused on parameter regimes where either a semiclassical interpretation of mesons in terms of domain walls is possible or a formulation as a simple gauge theory is amenable [54].

Alternatively, meson states can occur also far away from semiclassical regimes, e.g., close to quantum critical points (QCPs), where an effective (i.e., relativistic) quantum field theory (QFT) description is available. Zamolodchikov's $E_{8}$ model [55] is such an example of an interacting QFT that emerges through longitudinal perturbations at the Ising critical point. The theoretically predicted $E_{8}$ meson spectrum was first 
experimentally observed in [56] and found recently renewed interest in [57-59]. These experiments were based on neutron scattering measurements and spectroscopic methods in solidstate crystals.

Here, we instead propose controlled measurements of the $E_{8}$ meson spectrum on an ion-trap quantum simulator using absorption spectroscopy $[14,16]$. For that purpose, we numerically explore the capabilities of experimentally realizable small Ising spin systems to identify the lowest $E_{8}$ meson states. We show that for sufficiently strong long-range suppression in Ising models, the energy absorption spectrum, which is accessible in the linear response framework, is in close correspondence with the analytical expectation of the $E_{8}$ QFT. We corroborate these findings by a fidelity analysis, which suggests that small systems retain the nature of the first meson across all interaction ranges considered, while the thermodynamic limit may have a transition at a spatial power-law interaction $\sim 1 / r$. Due to the promising experimental [24,25] and theoretical [60-66] efforts to implement and study gauge theories with ion-trap quantum simulations, we see, as an implication of our study, the potential to probe meson physics also in relativistic gauge theories with these technologies.

\section{ISING MODELS AND QFTs}

The transverse field Ising model is a famous example of a many-body system exhibiting a quantum phase transition [67]. An additional longitudinal field can break the integrability of the system and introduces interesting new features, in particular mesons, appearing as nonperturbative bound states in the spectrum of the model. The prototype is the nearestneighbor (NN) Ising model, defined in terms of Pauli matrices $\sigma_{j}^{x, z}$ by the Hamiltonian

$$
H_{\mathrm{NN}}=-J\left(\sum_{j=1}^{N-1} \sigma_{j}^{z} \sigma_{j+1}^{z}+h \sum_{j=1}^{N} \sigma_{j}^{x}+g \sum_{j=1}^{N} \sigma_{j}^{z}\right),
$$

where the overall energy scale is set by the unit $J$. The transverse and longitudinal fields are quantified by the parameters $h$ and $g$, respectively. The Hamiltonian (1) is written for $N$ spins at positions $j$ assuming open boundary conditions (obc). Analogously, one can assume periodic boundary conditions (pbc), defined by $\sigma_{N+1}=\sigma_{1}$ for a system on a circle, by adding the interaction term $-J \sigma_{N}^{z} \sigma_{1}^{z}$.

In a proper continuum limit, the IR regime of $H_{\mathrm{NN}}$ is described by a Majorana fermion QFT with Hamiltonian [68]

$$
\begin{aligned}
H_{\mathrm{IR}}= & \int_{-\infty}^{\infty} d x\left\{\frac{i}{4 \pi}\left(\psi \partial_{x} \psi-\bar{\psi} \partial_{x} \bar{\psi}\right)\right. \\
& \left.-\frac{i M_{h}}{2 \pi} \bar{\psi} \psi+\mathcal{C} M_{g}^{15 / 8} \sigma\right\} .
\end{aligned}
$$

Here, $\mathcal{C} \approx 0.062$ is a numerical constant, and $M_{h} \equiv 2 J|1-h|$ and $M_{g} \equiv \mathcal{D} J|g|^{8 / 15}$ with $\mathcal{D} \approx 5.416$ are mass scales in the transverse and longitudinal direction $[68,69]$. The QCP at $\{J=h=1, g=0\}$ translates into $M_{h}=M_{g}=0$, in which case the IR is governed by the Ising CFT with central charge $c=1 / 2$ and scalar primary operators $\epsilon=i \bar{\psi} \psi$ and $\sigma$ of dimensions $\Delta_{\epsilon}=1$ and $\Delta_{\sigma}=1 / 8$. For longitudinal relevant perturbations of the Ising CFT, i.e., $M_{h}=0, M_{g} \neq 0$, it is a remarkable prediction of Zamolodchikov that the resulting interacting $E_{8}$ QFT is also integrable and governed by the exceptional simple Lie algebra of rank 8 [55]. This QFT contains eight stable mesons-fermionic nonperturbative bound states-whose masses are known as tabulated in Table I in units of the lightest meson mass $M_{1} \equiv M_{g}$.

On ion-trap quantum simulators, it is experimentally possible to implement a long-range (LR) Ising model, defined by the Hamiltonian $[12,13,15]$

$$
H_{\mathrm{LR}}=-J\left(\sum_{i<j}^{N} \frac{1}{|i-j|^{\alpha}} \sigma_{i}^{z} \sigma_{j}^{z}+h \sum_{j=1}^{N} \sigma_{j}^{x}+g \sum_{j=1}^{N} \sigma_{j}^{z}\right),
$$

where the coefficient $\alpha$ quantifies the LR interaction of two spins at position $i$ and $j$ [70]. Similarly to the NN model, one can consider the system for obc and pbc, where in the latter case we assume that two spins at positions $i$ and $j$ interact along their minimal distance on the ring. For $\alpha \rightarrow \infty$, one recovers the NN Hamiltonian (1). While experimentally the range $0 \leqslant \alpha \leqslant 3$ is in principle accessible [71,72], it was observed, e.g., in [73] that already for $\alpha \approx 3$, the physics of the system can resemble closely the NN model.

\section{ENERGY AND ABSORPTION SPECTRA}

In what follows, we compare the ideal NN model with the LR model based on numerical diagonalization, to characterize in how far the $E_{8}$ meson spectrum survives in the presence of power-law interactions and for the relatively small systems of a few dozen sites to which current experiments on trapped ions are restricted $[9,10]$. The basis for the observability in small systems is that the longitudinal field is chosen large enough such that the associated length scale of the first meson $L \sim 1 / M_{1} \sim|g|^{-8 / 15}$ is sufficiently small to be captured by the finite size chain. As already observed in [74] for a realistic model of a solid state crystal, even relatively large longitudinal field values are able to reproduce the $E_{8}$ spectrum, indicating the strong impact of the QFT regime on the physics of the model.

\section{A. Energy levels}

In Fig. 1, the mass gaps $m_{n} / m_{1}$ for the lowest $n=$ $1, \ldots, 400$ excited eigenstates, normalized to the lowest excited numerical state, are shown for a chain of $N=12$ spins with an exemplary longitudinal field $g=3$ in the NN (a) and LR (b) Ising model [75]. In the finite size system, energy levels appear as bands in the spectrum. In the ideal NN model, pbc (shown as orange triangles) allow for a clean identification of the first 6 meson levels. Apart from an underestimation of the fourth level, the mass ratios agree well with the $E_{8}$ theory (shown as grey dashed lines). The first $n=1, \ldots, N$ eigenvalues can be associated to the the first meson level and follow the momentum dispersion relation in the first Brillouin zone. In contrast, while obc (blue circles) match particularly some of the higher meson levels, they do not satisfy the ratio of the first $E_{8}$ masses. We therefore focus in the following on a finite system with pbc on a ring and compare the results to the $E_{8}$ theory on an infinite line (cf. Table I), i.e., we neglect finite volume corrections given by Lüscher's formula [76]. 
TABLE I. Ratios of meson masses for the integrable interacting $E_{8}$ QFT [55], which provides an effective description of the nearestneighbor Ising model along longitudinal perturbations at its critical point.

\begin{tabular}{lccccccc}
\hline \hline & $M_{2} / M_{1}$ & $M_{3} / M_{1}$ & $M_{4} / M_{1}$ & $M_{5} / M_{1}$ & $M_{6} / M_{1}$ & $M_{7} / M_{1}$ & $M_{8} / M_{1}$ \\
\hline Analytical & $2 \cos \frac{\pi}{5}$ & $2 \cos \frac{\pi}{30}$ & $4 \cos \frac{7 \pi}{30} \cos \frac{\pi}{5}$ & $4 \cos \frac{2 \pi}{15} \cos \frac{\pi}{5}$ & $4 \cos \frac{\pi}{30} \cos \frac{\pi}{5}$ & $8 \cos ^{2} \frac{\pi}{5} \cos ^{\frac{7 \pi}{30}}$ & $8 \cos \frac{\pi}{5} \cos \frac{2 \pi}{15}$ \\
Numerical & 1.6180 & 1.9890 & 2.4049 & 2.9563 & 3.2183 & 3.8912 & 4.7834 \\
\hline \hline
\end{tabular}

At energies above $2 M_{1}$, multiparticle states exist and form a continuum. Although we do not have a continuum in a finite system, we can nevertheless identify the mass sum $M_{1}+M_{2}$ (shown as the lowest grey dotted line). Higher order mass sums are very close to some of the analytical $E_{8}$ mass ratios. The LR results for $\alpha=3$ (right panel) resemble the NN profile nearly identically for pbc and are slightly smeared out for obc, and therefore similarly allow one to identify the analytical meson mass ratios.

\section{B. Absorption spectra}

In recent years, methods have been developed to reveal spectra of interacting spin systems in trapped ions akin to neutron scattering in the solid state $[14,16]$. Such experimentally measurable absorption spectra can be computed within the framework of linear response theory [77]. Specifically, the mean energy absorption rate $\bar{Q}=\overline{\langle\partial H / \partial t\rangle}$ is proportional to the imaginary (dissipative) part $\chi^{\prime \prime}(\omega) \equiv \chi_{A A}^{\prime \prime}(\omega)$ of the susceptibility, which is given in general in the Lehmann representation by

$$
\begin{aligned}
\chi_{O A}^{\prime \prime}(\omega)= & \pi \sum_{n, m=0}^{2^{N}-1}\langle n|A| m\rangle\langle m|O| n\rangle\left(p_{n}-p_{m}\right) \\
& \times \delta\left[\omega-\left(E_{m}-E_{n}\right)\right] .
\end{aligned}
$$

(a)

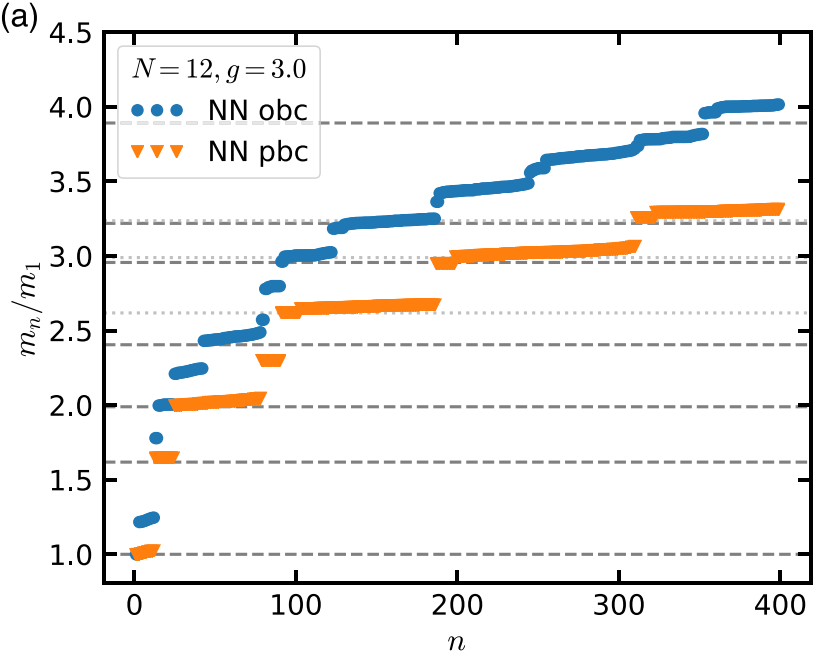

Here, the double sum is taken over all eigenstates $|n\rangle$ and $|m\rangle$ of the system, $A$ is an operator that perturbs the Hamiltonian in the time domain, and $O$ is an operator whose response in the system is considered. The delta function in Eq. (4) expresses the fact that there is only a contribution to the result when the perturbation frequency $\omega$ equals the energy differences $E_{m}-E_{n}$. For general thermal states, the population factors take the form $p_{n}=e^{-\beta E_{n}} / Z$, where $Z=\sum_{n} e^{-\beta E_{n}}$ is the finite temperature partition function. For our studies, we are interested in the zero temperature case where absorption energies are measured with respect to the ground state $|0\rangle$ with energy $E_{0}$, and $p_{0}=1$ and $p_{n}=0$ for $n>0$.

In the following, we find that the salient features of the spectrum become accessible with the following straightforwardly measurable operator:

$$
O=A=\sum_{i=1}^{N} \sigma_{i}^{z} \cos \left(k r_{i}\right),
$$

where $k \in[-\pi, \pi]$ is the quasimomentum and $r_{i}=a i \equiv i$ the lattice position for unit lattice spacing. For the special case of $k=0$, the imaginary part dynamic susceptibility simplifies (in dimensionless units) to

$$
\begin{aligned}
\chi^{\prime \prime}(\omega, k=0)= & \pi J \sum_{n=0}^{2^{N}-1} \sum_{i=1}^{N}\left|\left\langle 0\left|\sigma_{i}^{z}\right| n\right\rangle\right|^{2}\left\{\delta\left[\omega-\left(E_{n}-E_{0}\right)\right]\right. \\
& \left.-\delta\left[\omega+\left(E_{n}-E_{0}\right)\right]\right\} .
\end{aligned}
$$

(b)

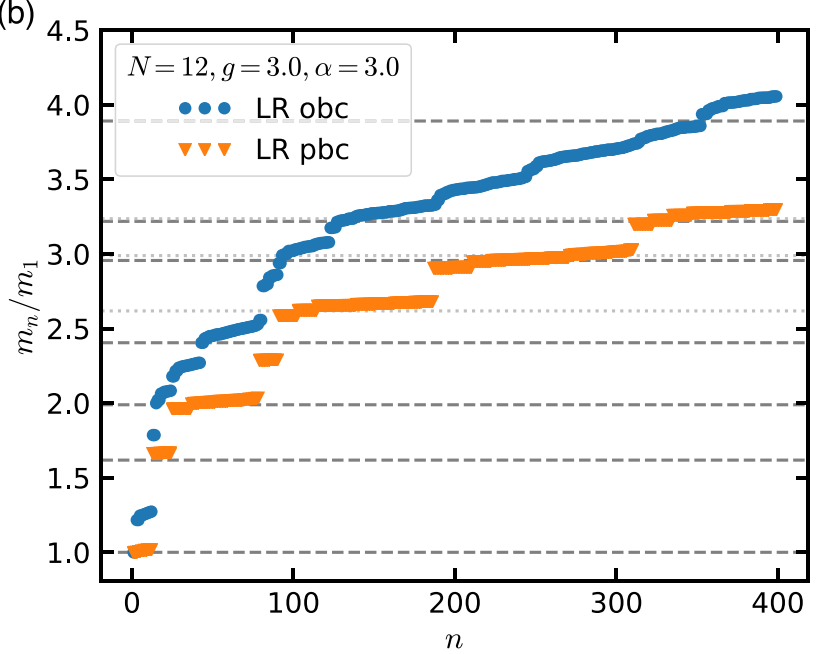

FIG. 1. Numerical energy spectra for the NN (a) and LR (b) Ising model with obc (blue circles) and pbc (orange triangles). The normalized mass gaps $m_{n} / m_{1}$ of the lowest excited states with level $n \in \mathbb{N}$ are shown for the longitudinal field strength $g=3$ in a chain of $N=12$ sites. The transverse field strength is fixed to the critical value $h=1$ throughout this work. Grey dashed lines represent the analytical $E_{8}$ meson mass ratios $M_{n} / M_{1}$ (cf. Table I). The continuum threshold is at $2 M_{1}$. Grey dotted lines correspond to multiparticle states with masses $M_{1}+M_{2}$, $M_{1}+M_{3}$, and $2 M_{2}$ (in ascending order). While for obc some deviations from the ideal result appear, for pbc even such a small system reproduces well the expected low-lying mass spectrum, for $\mathrm{NN}$ as well as for algebraic interactions. 


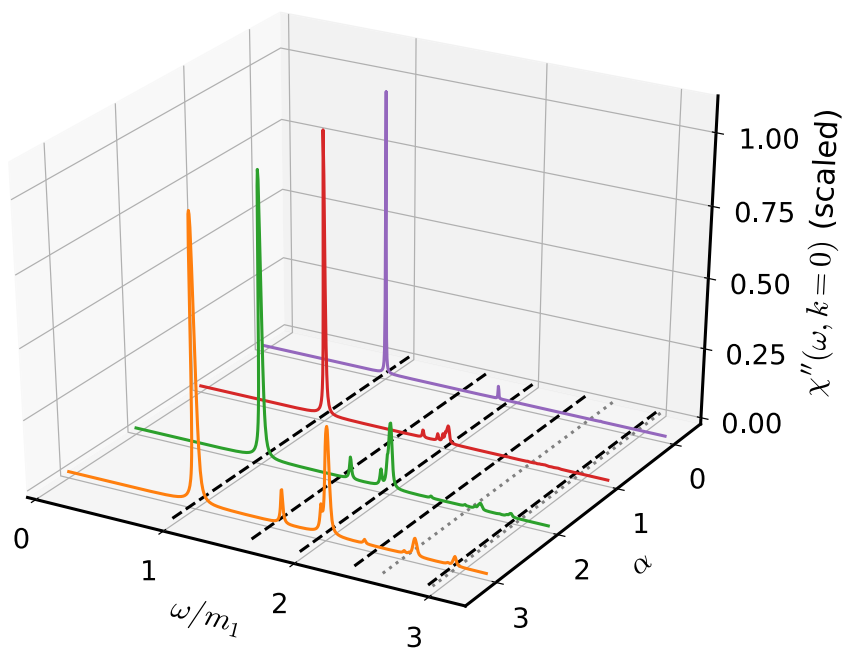

FIG. 2. Energy absorption spectrum of the LR model with pbc in dependence on the power-law coefficient $\alpha$. The data are scaled to the maximum of the spectrum. Black dashed lines represent the analytical $E_{8}$ meson mass ratios (cf. Table I). Grey dotted lines correspond to multiparticle states with masses $M_{1}+M_{2}$ and $M_{1}+M_{3}$. For the entire range of $\alpha$, a strong peak appears at the lowest meson mass. With increasing $\alpha$, more features become discernible that agree with the analytic $E_{8}$ meson spectrum of the QFT at the critical point of the NN Ising model. Numerical parameters: $N=18(\mathrm{pbc}), \Gamma / J=0.1$, $g=3$.

In a realistic situation, the energy resolution is restricted by the accessible experimental observation time $t_{\mathrm{obs}}$. According to the Wiener-Khintchine theorem [78,79], the delta function is then approximated by a Lorentzian

$$
\delta\left[\omega-\left(E_{n}-E_{0}\right)\right] \approx \frac{\Gamma}{\left[\omega-\left(E_{n}-E_{0}\right)\right]^{2}+\Gamma^{2}},
$$

with width $\Gamma=1 / t_{\mathrm{obs}}$.

We numerically calculate the energy absorption spectrum according to Eqs. (6) and (7) for the realistic value $\Gamma / J=0.1$ (see Sec. VI) on a chain of $N=18$ sites, which is the largest system size that we can achieve by iterative eigensolvers for sparse matrices, while keeping a large portion of the spectrum [80]. Figure 2 shows the energy absorption spectrum in the LR model as a function of the frequency in dependence of the coefficient $\alpha$. For low $\alpha$, only the first meson mass can unequivocally be discerned. As $\alpha$ is increased, peaks at the analytical $E_{8}$ meson mass ratios are formed, whereby the first meson retains the largest spectral density. The continuum threshold at $2 M_{1}$ overlaps with the third meson peak. Above, also the mass sum $M_{1}+M_{2}$ is identifiable while the fifth meson peak overlaps with the mass sum $M_{1}+M_{3}$.

In Fig. 3, the energy absorption spectrum in the LR model with $\alpha=3$ (green dash-dotted curve) is compared to the NN model (orange solid curve) for one selected value of the longitudinal field. The analogon of this spectral density in the $E_{8}$ QFT is the dynamical structure function, which has been calculated recently in [81]. The corresponding spectrum is shown as the blue dotted curve for a similar frequency broadening. In the Ising model data, the first five meson states and the mass sum $M_{1}+M_{2}$ are visible as peaks with (apart from the fourth level) good quantitative agreement to the analytical

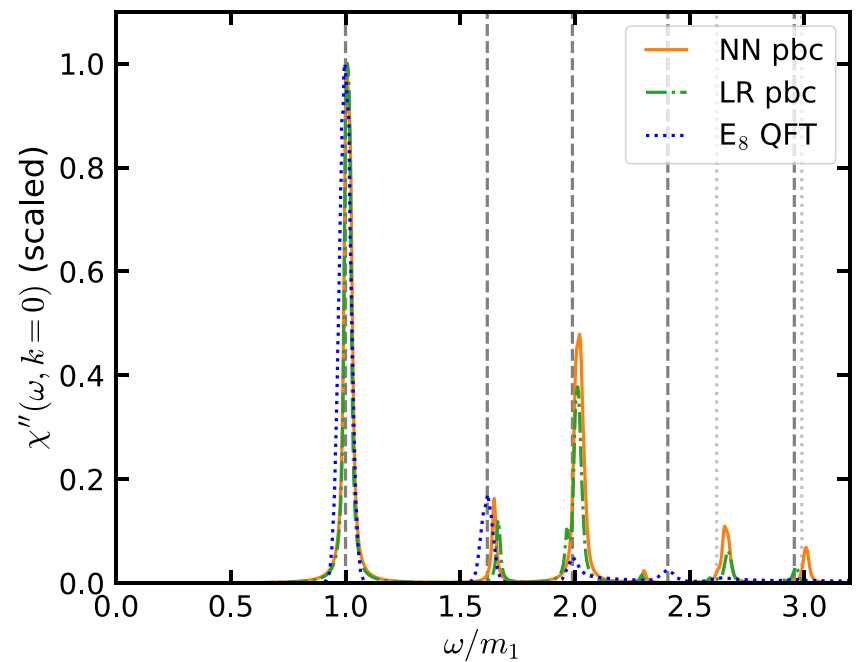

FIG. 3. Comparison of the energy absorption spectrum in the NN and LR models with the analytical $E_{8}$ dynamical structure function from [81]. The data are scaled to the maximum of the spectrum. Grey dashed lines represent the analytical $E_{8}$ meson mass ratios (cf. Table I). Grey dotted lines correspond to multiparticle states with masses $M_{1}+M_{2}$ and $M_{1}+M_{3}$. From the numerical absorption spectra, meson peaks can be identified very close to their expected analytical ratios. The LR model allows one to resolve the quantitative ratio of the first to the second meson peak height of the QFT prediction with nearly the same precision as the NN model. Numerical parameters: $N=18$ (pbc), $\Gamma / J=0.1, g=3, \alpha=3$.

mass ratios. While in the exact $E_{8}$ spectrum the meson peak heights are continuously decreasing, the finite size data are not able to reproduce this feature above the continuum threshold. However, the ratio of the first to the second meson peak height is even in good quantitative agreement with the analytical prediction.

\section{FIDELITY ANALYSIS}

The existence of a clear band structure in the energy spectrum of the LR Ising model (cf. Fig. 1) as well as peaks in the absorption spectrum (cf. Fig. 2) even for small values of $\alpha$ raises the question of whether the underlying quantum states still can be interpreted as mesons and whether they resemble their counterparts in the $E_{8}$ regime of the NN Ising model (corresponding to $\alpha=\infty$ ) for finite values of $\alpha$ even on the deeper level of quantum information measures. Regarding the first point, it is well known [28,29] that LR interactions confine domain walls in the Ising model. It is therefore in principle justified to interpret the existence of discrete band structures as meson states. In the present case, we have additionally also the effect of the longitudinal field. We address the resemblance with the $E_{8}$ regime of the $\mathrm{NN}$ model for this case using the fidelity $F(\alpha)$ and fidelity susceptibility $\chi_{F}(\alpha)$. These quantities have been used previously for ground $[82,83]$ and excited states $[84,85]$ as a theoretical framework to identify and characterize quantum phase transitions. Here, we use them to detect if there is a fundamental change in the meson structure as the LR coefficient $\alpha$ is varied. 



FIG. 4. Dependence of the fidelity (a), defined in (8), and fidelity susceptibility (b), defined in (9), on the LR coefficient $\alpha$. (a) In finite-size systems (colored solid lines, increasing $N$ from top to bottom), the first meson state retains a large overlap to the one of the $\mathrm{NN}$ model $(\alpha=\infty)$. An extrapolation to $N \rightarrow \infty$ (black dashed line) indicates a transition in the nature of the meson state in the thermodynamic limit, occurring at some value of $\alpha \lesssim 1.5$. (b) In agreement with this finding, the fidelity susceptibility shows a peak that becomes sharper with system size (colored solid lines, increasing $N$ from bottom to top). Assuming a scaling with $N^{-1}$, we obtain a peak position of $\alpha_{\max } \approx 1.07 \pm 0.02$ in the thermodynamic limit.

We consider the system with pbc, in which the first meson band consists of the first $n=1, \ldots, N$ excited eigenstates of the Hamiltonian. Since they have different degeneracies in the LR and NN model, the overlap of some of these states is not well defined and hence numerically not unique. In fact, only the first excited state $(n=1)$ is nondegenerate in all cases and allows us to define the fidelity as

$$
F(\alpha)=\left|\left\langle\phi_{1}(\alpha) \mid \phi_{1}(\alpha=\infty)\right\rangle\right|
$$

where $\phi_{1}$ denotes the first excited state in the LR and NN model, respectively. Furthermore, following $[82,83]$ the fidelity susceptibility is defined as

$$
\chi_{F}(\alpha)=-\left.\frac{\partial^{2} F(\alpha, \delta \alpha)}{\partial(\delta \alpha)^{2}}\right|_{\delta \alpha=0}=\lim _{\delta \alpha \rightarrow 0} \frac{-2 \ln F(\alpha, \delta \alpha)}{(\delta \alpha)^{2}},
$$

where $F(\alpha, \delta \alpha)=\mid\left\langle\phi_{1}(\alpha) \mid \phi_{1}(\alpha+\delta \alpha)\right\rangle$. In our numerics, we use the second relation with the numerical value $\delta \alpha=0.01$ and probe the range $0 \leqslant \alpha \leqslant 3$.

The results for the fidelity per site $f(\alpha) \equiv F(\alpha)^{1 / N}$ and the fidelity susceptibility $\chi_{F}(\alpha)$ are shown in Fig. 4 for several chain lengths at the longitudinal field value $g=3$. For all finite system sizes under consideration (colored solid curves), which are within experimental scope, for $\alpha \gtrsim 2, f(\alpha)$ lies close to the maximal value of 1 , and even at all-to-all LR interactions ( $\alpha=0$, lowest solid curve) the fidelity per site decreases at most by $1 \%$. These findings indicate that the quantum nature of the first excited state in the LR model resembles very closely its counterpart in the $\mathrm{NN}$ case, at least for finite system sizes.

In addition to the finite-size results, we extrapolate the data to the thermodynamic limit $N \rightarrow \infty$ by making the scaling ansatz $f(\alpha)=f_{\infty}(\alpha)+c(\alpha) N^{-b(\alpha)}$. We leave the exponent $b(\alpha)$ together with $c(\alpha)$ and $f_{\infty}(\alpha)$ as free fit parameter. The result for $f_{\infty}(\alpha)$, representing the prediction for the thermodynamic limit, is shown as the black dashed curve in the left panel of Fig. 4. While it shows fast convergence for large $\alpha$, it decreases rapidly for $\alpha \lesssim 1.5$, indicating a transition in the nature of the meson state for large system sizes. Similarly, the fidelity susceptibility, shown in the right panel, exhibits a peak at small values of $\alpha$ and then decreases towards 0 for strong LR suppression. Such a peak is suggestive of a transition in the first excited meson state occurring at some intermediate value of $\alpha$. As the system size increases, the peak position $\alpha_{\max }$ moves towards larger values of $\alpha$. Assuming a scaling with $N^{-1}$, we can extract the value $\alpha_{\max } \approx 1.07 \pm 0.02$ for $N \rightarrow \infty$ in the thermodynamic limit. This range seems to agree with the rapid decrease of the fidelity in the left panel.

Thus, while finite size systems retain the same physics across all considered values of $\alpha$, the scaling analysis suggest the appearance of interesting new physics for the first meson state in the LR versus NN model, which would be worthwhile to study on its own. This finding agrees with the fact that the Ising model with variable-range interactions in a transverse field shows a transition in its quench dynamics at $\alpha=1$ $[73,86]$. Besides hinting at interesting physics in the excited states, this result suggests that the mesons for at least $\alpha \gtrsim 1$ remain smoothly connected to the NN meson even at large system sizes.

\section{MESON MASS IDENTIFICATIONS}

For the previous discussions, one specific longitudinal field value was chosen. In this section, we extract the meson masses from the energy absorption spectrum in dependence of $g$ [87]. The results are presented in Fig. 5. Individual meson masses $\widetilde{M}_{n}$ are obtained from a Gaussian fit to each peak in units of the mass gap $m_{1}$ of the first excited state with an uncertainty corresponding to its full width at half maximum [shown in (a)]. Since the individual energy of an eigenstate is experimentally not accessible, we additionally express the results with respect to the extracted mass $\widetilde{M}_{1}$ of the first meson by propagating its uncertainty [shown in (b)]. With increasing value of $g$, the uncertainty of the meson mass decreases, allowing for a more 

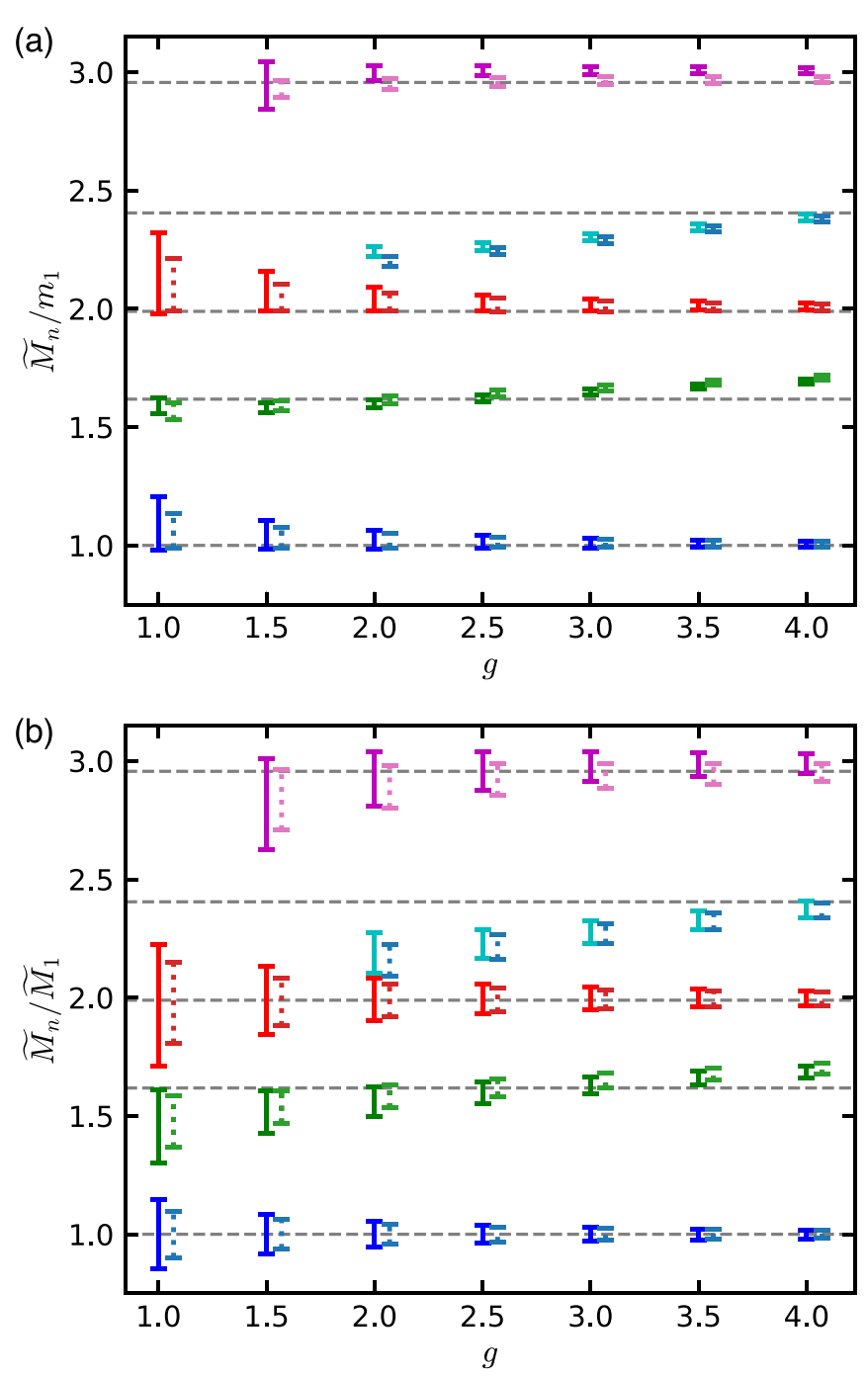

FIG. 5. Extracted meson mass ratios $\widetilde{M}_{n} / m_{1}$ and $\widetilde{M}_{n} / \widetilde{M}_{1}$ from the energy absorption spectra in dependence of the longitudinal field $g$. The results are expressed in units of the mass gap $m_{1}$ (a) and the first extracted meson mass $\widetilde{M}_{1}$ (b). The level $n=1,2, \ldots, 5$ increases from bottom to top. Solid error bars are for the NN model, dotted ones for the LR model (shown slightly displaced for graphical purposes). Grey dashed lines represent the the analytical $E_{8}$ meson mass ratios $M_{n} / M_{1}$ (cf. Table I). Numerical parameters: $N=18$ (pbc), $\Gamma / J=0.1, \alpha=3$. Once $g$ is sufficiently large, the meson mass ratios can be reliably extracted even in small systems.

precise identification of the analytical $E_{8}$ mass ratios $M_{n} / M_{1}$ up to the fifth level for both the NN (solid error bars) and the LR model (dotted error bars). The fourth meson is constantly underestimated except for the largest considered longitudinal field strength. Overall, the numerical data of the finite size system are closest to the $E_{8}$ QFT in the range $3 \leqslant g \leqslant 4$, with an even smaller uncertainty for the LR model. Moreover, one can observe that the measurable ratio in panel (b) even allows for a slightly better consistency with the analytical $E_{8}$ mass ratios. The resulting uncertainties (peak widths) follow as a property of the spectrum in combination with the chosen inverse observation time $\Gamma / J=0.1$.

\section{QUANTUM SIMULATION IN TRAPPED IONS}

In trapped-ion quantum simulators, effective magnetic models are routinely realized by encoding the basis states $|\uparrow\rangle$ and $|\downarrow\rangle$ of spins $1 / 2$ in two long-lived hyperfine states and inducing effective spin-spin interactions $\sim J$ through a phonon bus, e.g., using a Mølmer-Sørensen-type laser or microwave beam [88]. Effective magnetic fields $\sim h, g$ can be realized by a detuning of the Mølmer-Sørensen beams [13,15,16,89,90] or by additional lasers that are tuned off-resonantly to the carrier transition [17,23].

An experimental protocol to measure the $E_{8}$ spectrum in such a system is as follows. First, the effective spins are prepared in the electronic ground state, corresponding to the fully polarized state $|\uparrow, \ldots, \uparrow\rangle$, the ground state at $g=\infty$. By slowly decreasing $g$ and turning on $J$ and $h$, the system is adiabatically transferred to the ground state at the desired parameter values. Such a procedure can produce considerable excitations when crossing a quantum phase transition [13]. In the present scenario, instead, even though we are interested in the QFT regime emanating from the critical point, we find the final value of the transverse field $g$ can be large, ensuring a large many-body gap on the order of $M_{g} \equiv \mathcal{D} J|g|^{8 / 15}$. For $g=3$, we have $M_{g}=9.7 \mathrm{~J}$ and thus the initial state preparation can occur adiabatically in times much shorter than $\hbar / J$, which in turn are much shorter than typical coherence times. Alternatively, ground states in trapped-ion quantum computers can be prepared to good precision using variational algorithms [25].

After initialization, the system is perturbed with a timedependent magnetic field, which again can be realized by periodically modulating the detuning of the MølmerSørensen beams or by a time-modulated ac-Stark shift. Using single-site addressing, site dependent ac-Stark shifts with switching times much faster than the timescales of the internal dynamics (on the order of $\hbar / J$ ) have already been demonstrated experimentally $[17,23]$. It is thus possible to perturb the effective spin system with an operator of the type defined in Eq. (5).

Two spectroscopy protocols are thinkable: The perturbation is turned on abruptly, e.g., as a step function and the subsequent time evolution of the same observable is tracked, which for local observables of the type $O$ can be done by standard fluorescence measurements [9,10,91]. A Fourier transform then yields the desired imaginary part of the dynamic susceptibility, $\chi^{\prime \prime}(\omega)$, defined in Eq. (6) [92]. Alternatively, the perturbation can be modulated temporally with a $\cos (\omega t)$. By tracking the absorbed energy per unit time, which amounts to the measurement of few-body correlators and which has already been demonstrated experimentally [25], again $\chi^{\prime \prime}(\omega)$ is obtained.

Typical trapped-ion experiments on many-body spin systems generate long-range interactions [12-16]. In linear chains with open boundary conditions, for not too large systems these approximate a spatial power-law decay to good precision [72,93], and any deviations from the desired powerlaw interactions can be mitigated by the shaping of the interactions, e.g., by additional laser beams [63,90,94], periodic driving [95,96], or trap-shaping techniques [61,9799]. It is nowadays also possible to prepare ions in ring 


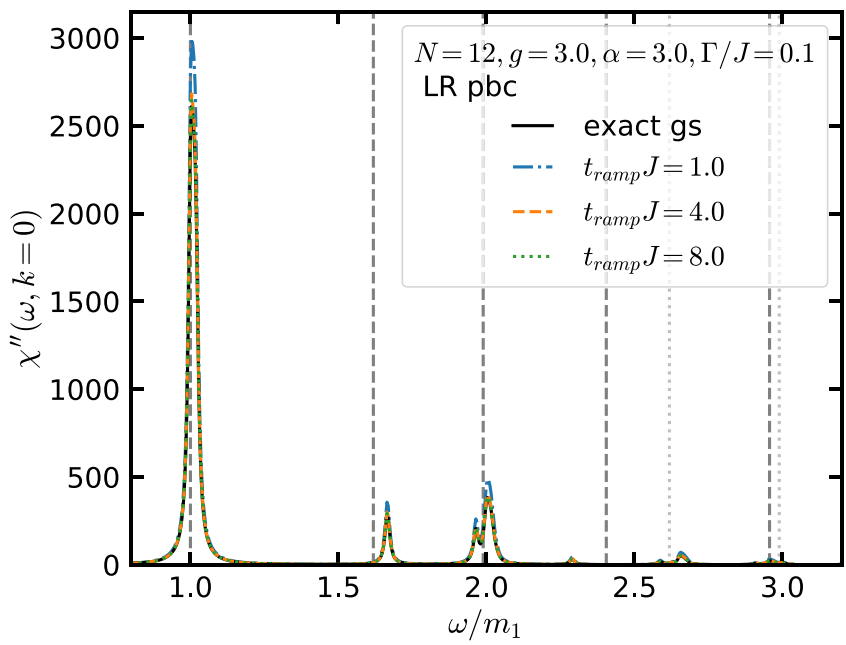

FIG. 6. Comparison of the energy absorption spectra for adiabatically prepared ground states with different time durations $t_{\text {ramp }}$ of a linear ramp (colored dashed curves) to the exact ground state case in the LR model (black solid curve). Background lines are as in Fig. 3. Even for a relatively fast ramp of $t_{\mathrm{ramp}} J=1$, the qualitative agreement of the spectrum with the exact case is very good and allows for the identification of $E_{8}$ meson mass ratios.

conformations, thus enabling the realization of periodic boundary conditions [100-102]. While the theoretical range of power-law decay exponents is $0 \leqslant \alpha \leqslant 3$ [71], the experimentally most favorable power-law decays are at $\alpha=0$ when working with the axial center-of-mass mode or in an intermediate range when interactions are transmitted by the radial phonon modes. For example, in [15] the range $0.75 \leqslant$ $\alpha \leqslant 1.75$ has been accessed, which, as the spectra and fidelity analysis reported in the previous sections show, enables access to meson spectra that closely approach the physics of the ideal NN model.

The robustness of the adiabatic ground state preparation and subsequent spectroscopic analysis is explicitly demonstrated in Fig. 6 for the LR model. Here, we consider the previously discussed parameters $\alpha=g=3$ for a small chain of $N=12$ sites. Starting from the fully polarized state, the ground state in the $E_{8}$ regime is adiabatically prepared by increasing the transverse field from $h=0$ up to $h=1$ using a linear ramp profile of duration $t_{\text {ramp }}$. We assume $J d t=0.01$, from which $d h$ follows through the relation $d h / d t=1 / t_{\text {ramp }}$. The figure shows the resulting absorption spectra for several values of $t_{\text {ramp }} \lesssim M_{g}$ (colored dashed curves) in comparison to the case when the exact ground state is calculated and employed in Eq. (6) (black solid curve). The curves for $t_{\text {ramp }} J=\{4,8\}$ are nearly coinciding with the exact result. Only for a fast ramp with $t_{\text {ramp }} J=1$ the peak heights are marginally overestimated but still allow for a precise meson identification.

\section{SUMMARY AND OUTLOOK}

In this article, we have demonstrated that the relativistic $E_{8}$ QFT can be identified experimentally on ion-trap quantum simulators. Surprisingly small systems of only 12 to 18 sites with pbc, which implement the LR quantum Ising model at the experimentally largest possible LR suppression, resemble the NN Ising model closely and allow for the identification of $E_{8}$ meson states. For longer-ranged interactions, while most meson states disappear from the spectrum, the lowest meson remains a strong feature. We have calculated the energy absorption spectrum based on linear response theory and showed that it shares qualitative and quantitative features with its QFT counterpart. Single and multiparticle meson states appear as peaks in the energy absorption spectrum, which allow for a precise extraction of analytically predicted $E_{8}$ meson mass ratios even for large longitudinal field values. As a fidelity analysis shows, for small systems the nature of the first meson changes only insignificantly across all values of $\alpha$ considered, while we find indications for a transition in the meson state in the thermodynamic limit at a critical value around $\alpha_{c} \approx 1$. We have also discussed a protocol adapted to existing trappedion technologies to experimentally access the meson spectra. While we have focused on the zero momentum case, this procedure can be extended to derive also relativistic dispersion relations at finite momenta, which would enable us, e.g., to extract the meson dispersion relation.

We have focused in our study on the $E_{8}$ regime, which appears in a parameter region of the simple Ising model (longitudinal perturbations at the QCP) and has been experimentally verified previously in solid state crystals. Using ion-trap based quantum simulation technologies opens, however, a new avenue to address relativistic meson physics also in more complicated gauge theories; see, e.g., [103] for an overview of recent progress in the field. Furthermore, ion-trap quantum simulations allow for studies of finite-temperature systems [104,105], which offers, for example, the possibility to study the rich physics of meson melting [106], a process for which currently no complete microscopic understanding is available [107].
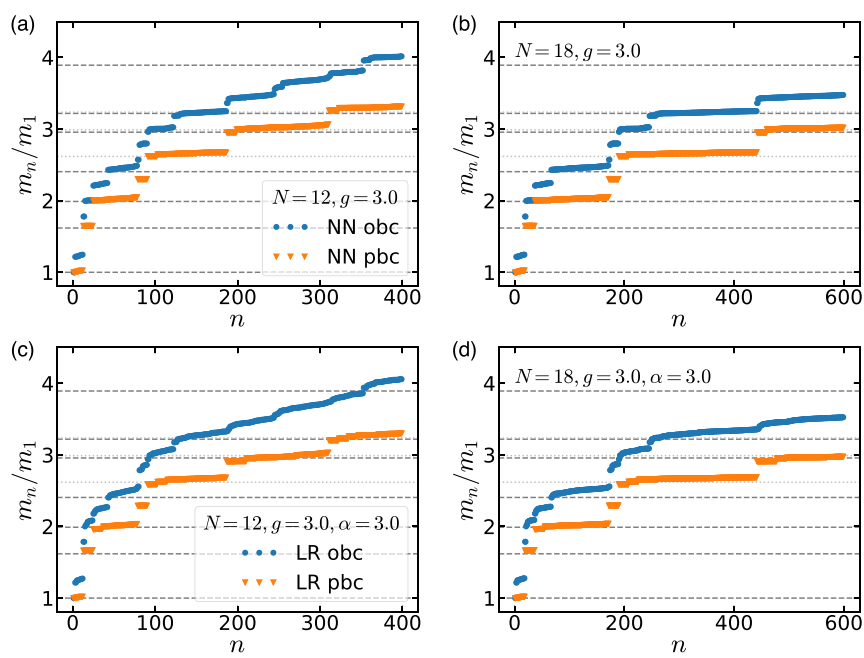

FIG. 7. Numerical energy spectra (normalized mass gaps of excited states) for the NN (a),(b) and LR (c),(d) Ising model with obc (blue circles) and pbc (orange triangles). The left column is for a chain of size $N=12$, the right for $N=18$. Grey dashed lines represent the the analytical $E_{8}$ meson mass ratios (cf. Table I). Grey dotted lines correspond to multiparticle states with masses $M_{1}+M_{2}$, $M_{1}+M_{3}$, and $2 M_{2}$ (in ascending order). 

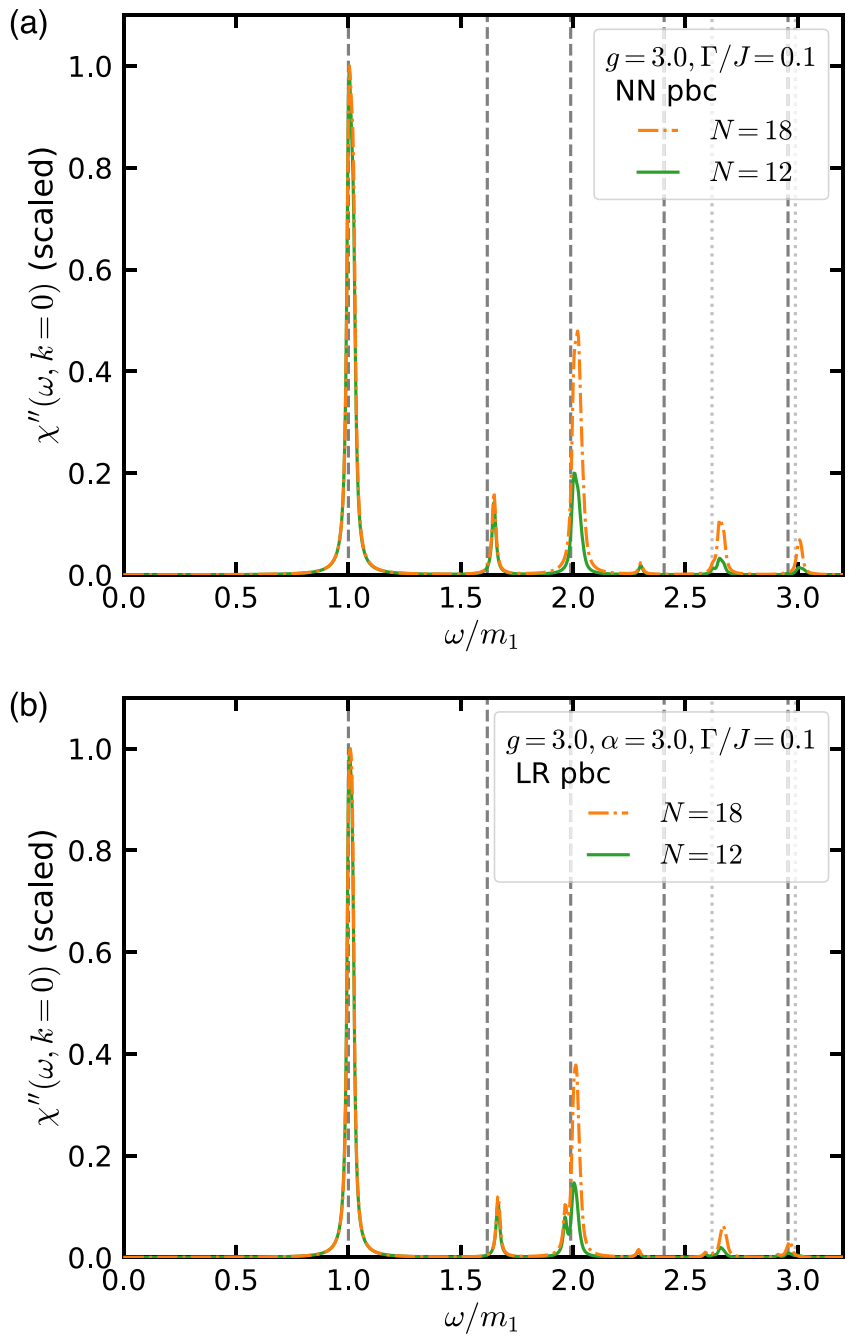

FIG. 8. Comparison of the energy absorption spectrum in the NN (a) and LR Ising model (b) for different chain sizes $N$. The data are scaled to the maximum of the spectrum. Grey dashed lines represent the the analytical $E_{8}$ meson mass ratios (cf. Table I). Grey dotted lines correspond to multiparticle states with masses $M_{1}+M_{2}$ and $M_{1}+M_{3}$.

\section{ACKNOWLEDGMENTS}

The Gravity, Quantum Fields and Information group at AEI was generously supported by the Alexander von Humboldt Foundation and the Federal Ministry for Education and Research through the Sofja Kovalevskaja Award. J.K. was partially supported by the International Max Planck Research School for Mathematical and Physical Aspects of Gravitation, Cosmology and Quantum Field Theory. The work of J.K. was supported in part by a fellowship from the Studienstiftung des deutschen Volkes (German Academic Scholarship Foundation). P.H. was supported by Provincia Autonoma di Trento, the ERC Starting Grant StrEnQTh (Project No. ID 804305), the Google Research Scholar Award ProGauge, and Q@TN_Quantum Science and Technology in Trento.
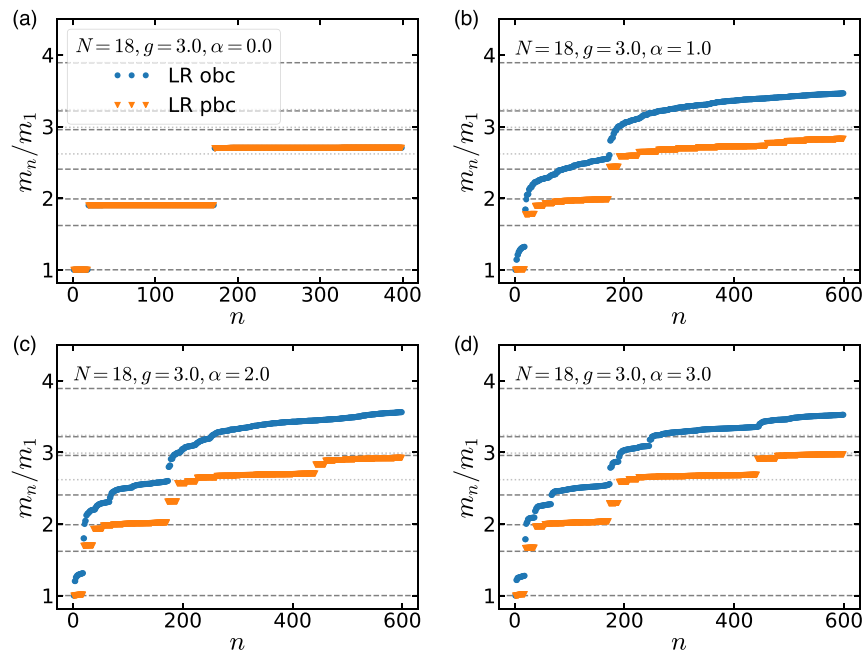

FIG. 9. Effect of the LR coefficient $\alpha$ on the numerical energy spectrum (normalized mass gaps of excited states) with obc (blue circles) and pbc (orange triangles). Background lines are as in Fig. 7.
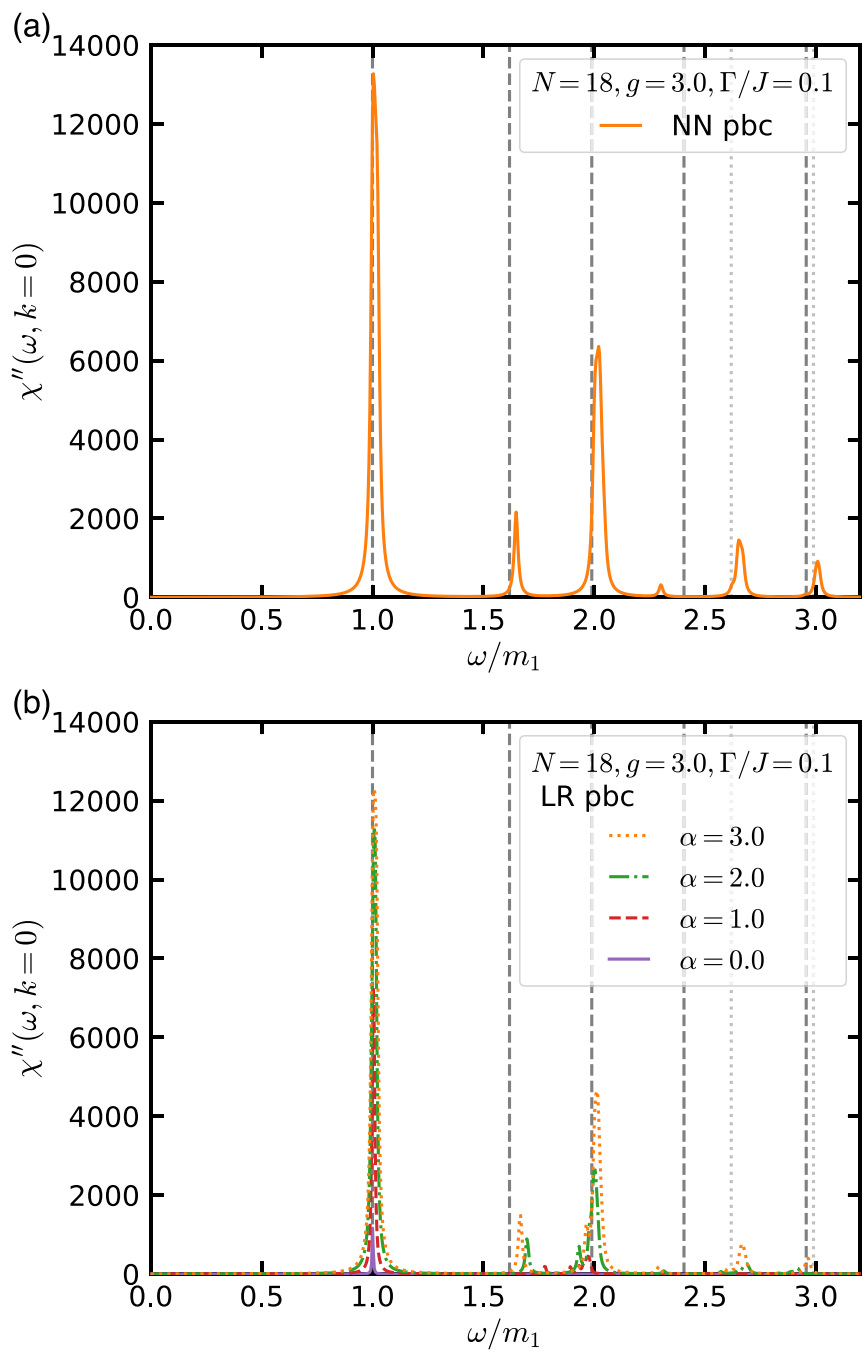

FIG. 10. Comparison of the absorption spectrum in the $\mathrm{NN}$ (a) and LR Ising model (b). In the LR model, the coefficient $\alpha$ is varied over the whole experimentally accessible range of values. Background lines are as in Fig. 8. 

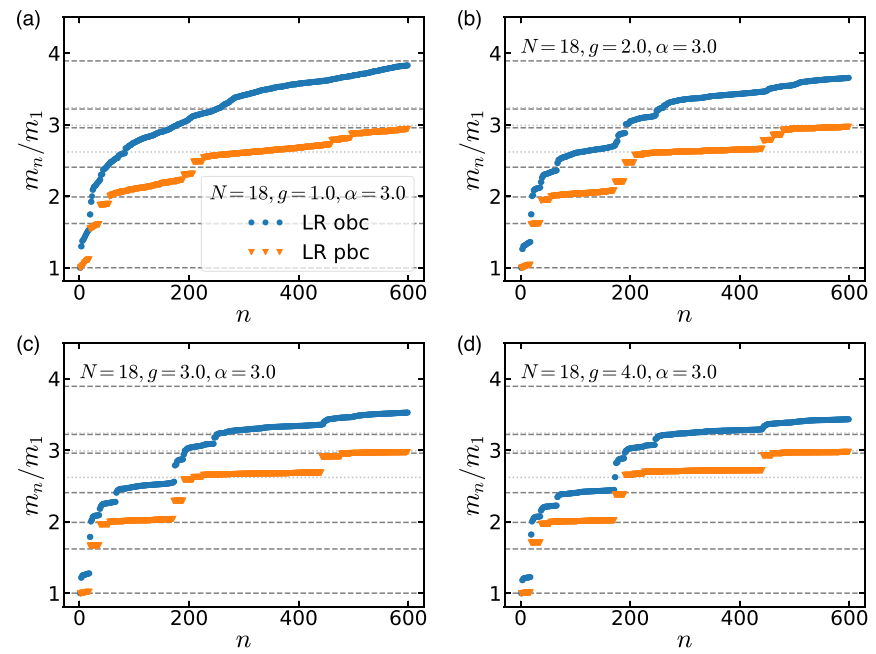

FIG. 11. Effect of the longitudinal field value $g$ on the numerical energy spectrum (normalized mass gaps of excited states) in the LR model with obc (blue circles) and pbc (orange triangles). Background lines are as in Fig. 7.

\section{APPENDIX A: FINITE SIZE EFFECTS}

In this Appendix we elaborate on some further details about the finite size dependence of the results presented in the main text.

(a)
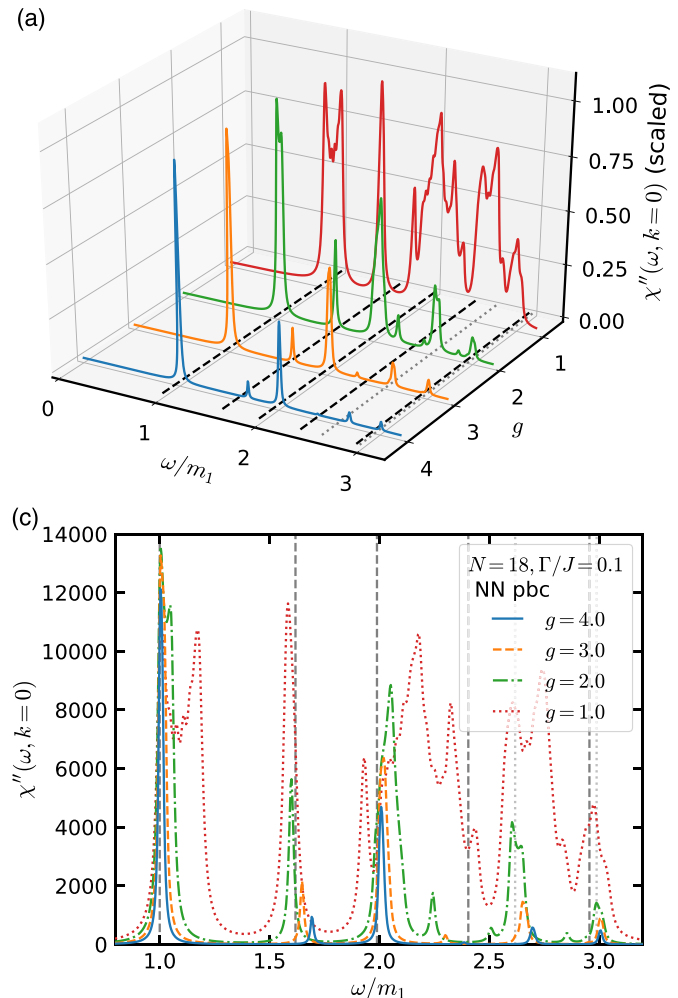

In Fig. 7, the normalized mass gaps of a chain of size $N=12$ [left column (a),(c) as in Fig. 1 of the main text] are compared to a chain with $N=18$ sites [right column (b),(d)]. The longitudinal field $g=3$ and the largest experimentally accessible decay parameter $\alpha=3$ are considered. Due to the exponential difference in the total number of eigenstates, different portions of the spectrum are available for a comparable number of excited states. For both obc and pbc, the effect of the finite size difference seems to be very mild in the energy spectrum. Observe also that for obc, higher bands in the LR model seem to resemble a continuous branch. There are only mild differences between the NN model [top row (a),(b)] and the LR model at $\alpha=3$ [bottom row (c),(d)].

The underlying eigenstates give rise to the energy absorption spectra shown in Fig. 8. There are nearly no visible differences for the first two meson peaks. Only above the continuum threshold, differences in multiparticle states occur. We therefore conclude that the quantitative agreement with the analytical $E_{8}$ result for the dynamical structure function, which is described in the main text, is a stable feature for both the NN and LR model.

\section{APPENDIX B: LONG-RANGE DEPENDENCE}

In this Appendix, we analyze the effect of the LR coefficient $\alpha$ on the physics discussed in the main text.

Figure 9 displays the energy spectrum as a function of $\alpha$. We vary the parameter in the range $\alpha=0$ (all-to-all LR
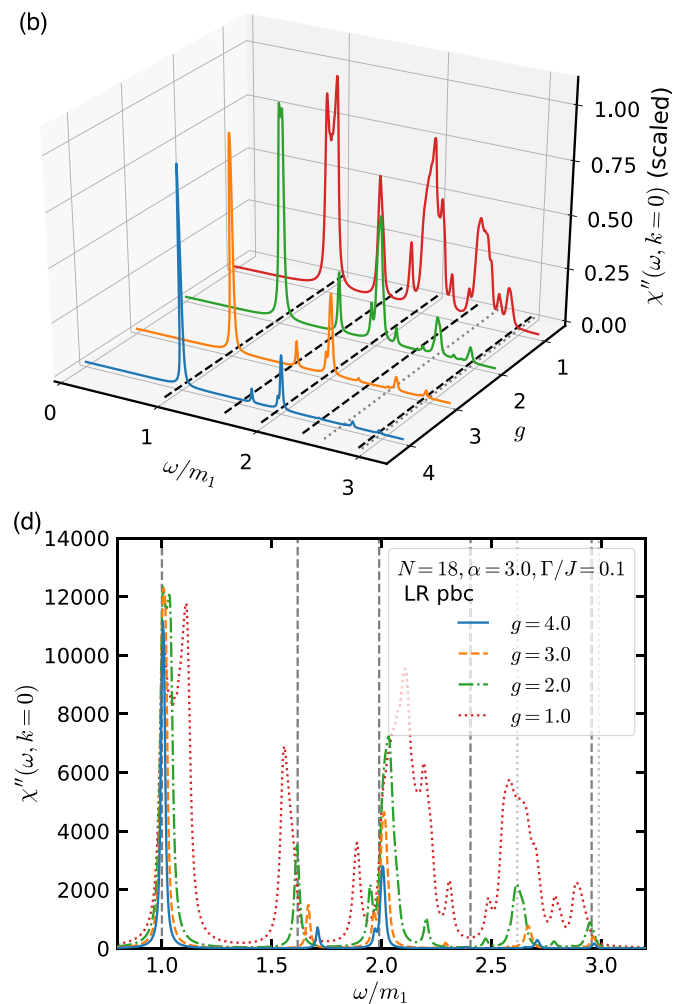

FIG. 12. Energy absorption spectrum of the NN (a),(c) and LR model (b),(d) with pbc in dependence on the longitudinal field value $g$. In (a),(b) the data are scaled to the maximum of the spectrum, in (c),(d) in absolute units. Dashed lines represent the analytical $E_{8}$ meson mass ratios (cf. Table I), dotted lines correspond to multiparticle states with masses $M_{1}+M_{2}$ and $M_{1}+M_{3}$. Numerical parameters: $N=18$ (pbc), $\Gamma / J=0.1, \alpha=3$. 
interactions) up to the previously used $\alpha=3$ (strong LR suppression). At $\alpha=0$, three identical degenerate branches are visible within the considered portion of the spectrum, for obc and pbc. For increasing values of $\alpha$, semicontinuous branches in the obc spectrum are more and more split into discrete bands and new bands appear in the case of pbc. Already for $\alpha \geqslant 2$, the bands in the pbc spectrum resemble the $E_{8}$ lines.

The resulting energy absorption spectra are shown in Fig. 10(b) in absolute units for a quantitative comparison to the NN model (a). As $\alpha$ is increased, the intensities of the individual peaks are increased and the peak positions resemble the $E_{8}$ mass ratios. Observe that there is only a very small difference in the absolute height of the first peak for the NN versus the LR model at $\alpha=3$.

\section{APPENDIX C: LONGITUDINAL FIELD DEPENDENCE}

In this Appendix we provide further details about the longitudinal field dependence of the absorption spectra.
Figure 11 shows the energy spectra of the LR model at $\alpha=3$ for several longitudinal field values. With increasing field strength, it becomes visible that a large semicontinuous band breaks apart into several discrete bands, which flatten out at the expected analytical $E_{8}$ mass ratios for pbc.

The resulting energy absorption spectra are compared to the NN model in Fig. 12. For a better visual presentation, we show both the scaled spectra in the top panels (a),(b) as well in absolute units in the bottom panels (c),(d) for a quantitative comparison. With increasing longitudinal field, the peaks get narrower and allow for the identification of the proper meson mass ratios and sums. For the system size under consideration, $N=18$, one can infer that a longitudinal field $g \geqslant 2$ is necessary to capture all associated of the lowlying meson length scales in the system. The NN spectrum differs qualitatively from the LR model only at the smallest depicted field value $g=1$ (red curves). At larger values, the quantitative differences in the absolute scale are very small.
[1] P. W. Anderson, More is different, Science 177, 393 (1972).

[2] P. Coleman, Introduction to Many-Body Physics (Cambridge University Press, Cambridge, UK, 2015).

[3] E. Witten, Symmetry and emergence, Nat. Phys. 14, 116 (2018).

[4] P. Hauke, F. M. Cucchietti, L. Tagliacozzo, I. Deutsch, and M. Lewenstein, Can one trust quantum simulators? Rep. Prog. Phys. 75, 082401 (2012).

[5] J. I. Cirac and P. Zoller, Goals and opportunities in quantum simulation, Nat. Phys. 8, 264 (2012).

[6] Y. Alexeev et al., Quantum computer systems for scientific discovery, PRX Quantum 2, 017001 (2021).

[7] C. Schneider, D. Porras, and T. Schaetz, Experimental quantum simulations of many-body physics with trapped ions, Rep. Prog. Phys. 75, 024401 (2011).

[8] R. Blatt and C. F. Roos, Quantum simulations with trapped ions, Nat. Phys. 8, 277 (2012).

[9] C. Monroe, W. C. Campbell, L.-M. Duan, Z.-X. Gong, A. V. Gorshkov, P. W. Hess, R. Islam, K. Kim, N. M. Linke, G. Pagano, P. Richerme, C. Senko, and N. Y. Yao, Programmable quantum simulations of spin systems with trapped ions, Rev. Mod. Phys. 93, 025001 (2021).

[10] I. Pogorelov, T. Feldker, C. D. Marciniak, L. Postler, G. Jacob, O. Krieglsteiner, V. Podlesnic, M. Meth, V. Negnevitsky, M. Stadler, B. Höfer, C. Wächter, K. Lakhmanskiy, R. Blatt, P. Schindler, and T. Monz, Compact ion-trap quantum computing demonstrator, PRX Quantum 2, 020343 (2021).

[11] A. Friedenauer, H. Schmitz, J. T. Glueckert, D. Porras, and T. Schaetz, Simulating a quantum magnet with trapped ions. Nat. Phys. 4, 757 (2008).

[12] J. W. Britton, B. C. Sawyer, A. C. Keith, C.-C. J. Wang, J. K. Freericks, H. Uys, M. J. Biercuk, and J. J. Bollinger, Engineered two-dimensional ising interactions in a trapped-ion quantum simulator with hundreds of spins, Nature (London) 484, 489 (2012).

[13] P. Richerme, C. Senko, S. Korenblit, J. Smith, A. Lee, R. Islam, W. C. Campbell, and C. Monroe, Quantum Catalysis of Magnetic Phase Transitions in a Quantum Simulator, Phys. Rev. Lett. 111, 100506 (2013).

[14] C. Senko, J. Smith, P. Richerme, A. Lee, W. C. Campbell, and C. Monroe, Coherent imaging spectroscopy of a quantum many-body spin system, Science 345, 430 (2014).

[15] P. Jurcevic, B. P. Lanyon, P. Hauke, C. Hempel, P. Zoller, R. Blatt, and C. F. Roos, Quasiparticle engineering and entanglement propagation in a quantum many-body system, Nature (London) 511, 202 (2014).

[16] P. Jurcevic, P. Hauke, C. Maier, C. Hempel, B. P. Lanyon, R. Blatt, and C. F. Roos, Spectroscopy of Interacting Quasiparticles in Trapped Ions, Phys. Rev. Lett. 115, 100501 (2015).

[17] J. Smith, A. Lee, P. Richerme, B. Neyenhuis, P. W. Hess, P. Hauke, M. Heyl, D. A. Huse, and C. Monroe, Many-body localization in a quantum simulator with programmable random disorder, Nat. Phys. 12, 907 (2016).

[18] M. Gärttner, J. G. Bohnet, A. Safavi-Naini, M. L. Wall, J. J. Bollinger, and A. M. Rey, Measuring out-of-time-order correlations and multiple quantum spectra in a trapped ion quantum magnet, Nat. Phys. 13, 781 (2017).

[19] P. Jurcevic, H. Shen, P. Hauke, C. Maier, T. Brydges, C. Hempel, B. P. Lanyon, M. Heyl, R. Blatt, and C. F. Roos, Direct Observation of Dynamical Quantum Phase Transitions in an Interacting Many-Body System, Phys. Rev. Lett. 119, 080501 (2017).

[20] J. Zhang, G. Pagano, P. W. Hess, A. Kyprianidis, P. Becker, H. Kaplan, A. V. Gorshkov, Z.-X. Gong, and C. Monroe, Observation of a many-body dynamical phase transition with a 53-qubit quantum simulator, Nature (London) 551, 601 (2017).

[21] C. Hempel, C. Maier, J. Romero, J. McClean, T. Monz, H. Shen, P. Jurcevic, B. P. Lanyon, P. Love, R. Babbush et al., Quantum Chemistry Calculations on a Trapped-Ion Quantum Simulator, Phys. Rev. X 8, 031022 (2018).

[22] T. Brydges, A. Elben, P. Jurcevic, B. Vermersch, C. Maier, B. P. Lanyon, P. Zoller, R. Blatt, and C. F. Roos, Probing Rényi 
entanglement entropy via randomized measurements, Science 364, 260 (2019).

[23] C. Maier, T. Brydges, P. Jurcevic, N. Trautmann, C. Hempel, B. P. Lanyon, P. Hauke, R. Blatt, and C. F. Roos, EnvironmentAssisted Quantum Transport in a 10-Qubit Network, Phys. Rev. Lett. 122, 050501 (2019).

[24] E. A. Martinez et al., Real-time dynamics of lattice gauge theories with a few-qubit quantum computer, Nature (London) 534, 516 (2016).

[25] C. Kokail et al., Self-verifying variational quantum simulation of lattice models, Nature (London) 569, 355 (2019).

[26] B. Friman, C. Hohne, J. Knoll, S. Leupold, J. Randrup, R. Rapp, and P. Senger, The CBM physics book: compressed baryonic matter in laboratory experiments, Lect. Notes Phys. 814, 1 (2011).

[27] B. M. McCoy and T. T. Wu, Two-dimensional ising field theory in a magnetic field: breakup of the cut in the two point function, Phys. Rev. D 18, 1259 (1978).

[28] F. Liu, R. Lundgren, P. Titum, G. Pagano, J. Zhang, C. Monroe, and A. V. Gorshkov, Confined Quasiparticle Dynamics in Long-Range Interacting Quantum Spin Chains, Phys. Rev. Lett. 122, 150601 (2019).

[29] A. Lerose, B. Zunkovic, A. Silva, and A. Gambassi, Quasilocalized excitations induced by long-range interactions in translationally invariant quantum spin chains, Phys. Rev. B 99, 121112(R) (2019).

[30] M. Greiter, Fictitious flux confinement: Magnetic pairing in coupled spin chains or planes, Phys. Rev. B 66, 054505 (2002).

[31] B. Lake, A. M. Tsvelik, S. Notbohm, D. Alan Tennant, T. G. Perring, M. Reehuis, C. Sekar, G. Krabbes, and B. Büchner, Confinement of fractional quantum number particles in a condensed-matter system, Nat. Phys. 6, 50 (2009).

[32] M. Kormos, M. Collura, G. Takacs, and P. Calabrese, Real-time confinement following a quantum quench to a nonintegrable model, Nat. Phys. 13, 246 (2017).

[33] A. J. A. James, R. M. Konik, and N. J. Robinson, Nonthermal States Arising from Confinement in One and Two Dimensions, Phys. Rev. Lett. 122, 130603 (2019).

[34] P. P. Mazza, G. Perfetto, A. Lerose, M. Collura, and A. Gambassi, Suppression of transport in nondisordered quantum spin chains due to confined excitations, Phys. Rev. B 99, 180302(R) (2019).

[35] N. J. Robinson, A. J. A. James, and R. M. Konik, Signatures of rare states and thermalization in a theory with confinement, Phys. Rev. B 99, 195108 (2019).

[36] L. Vanderstraeten, E. Wybo, N. Chepiga, F. Verstraete, and F. Mila, Spinon confinement and deconfinement in spin- 1 chains, Phys. Rev. B 101, 115138 (2020).

[37] A. Lerose, F. M. Surace, P. P. Mazza, G. Perfetto, M. Collura, and A. Gambassi, Quasilocalized dynamics from confinement of quantum excitations, Phys. Rev. B 102, 041118(R) (2020).

[38] M. C. Banuls, M. P. Heller, K. Jansen, J. Knaute, and V. Svensson, From spin chains to real-time thermal field theory using tensor networks, Phys. Rev. Research 2, 033301 (2020).

[39] O. A. Castro-Alvaredo, M. Lencses, I. M. Szecsenyi, and J. Viti, Entanglement Oscillations near a Quantum Critical Point, Phys. Rev. Lett. 124, 230601 (2020).

[40] J. C. Halimeh, M. Van Damme, V. Zauner-Stauber, and L. Vanderstraeten, Quasiparticle origin of dynamical quantum phase transitions, Phys. Rev. Research 2, 033111 (2020).
[41] N. Defenu, T. Enss, and J. C. Halimeh, Dynamical criticality and domain-wall coupling in long-range Hamiltonians, Phys. Rev. B 100, 014434 (2019).

[42] J. C. Halimeh, M. V. Damme, L. Guo, J. Lang, and P. Hauke, Dynamical phase transitions in quantum spin models with antiferromagnetic long-range interactions, Phys. Rev. B 104, 115133 (2021).

[43] T. Hashizume, I. P. McCulloch, and J. C. Halimeh, Dynamical phase transitions in the two-dimensional transverse-field ising model, arXiv:1811.09275 (2020).

[44] T. Hashizume, J. C. Halimeh, and I. P. McCulloch, Hybrid infinite time-evolving block decimation algorithm for long-range multidimensional quantum many-body systems, Phys. Rev. B 102, 035115 (2020).

[45] W. L. Tan et al., Domain-wall confinement and dynamics in a quantum simulator, Nat. Phys. 17, 742 (2021).

[46] J. Vovrosh and J. Knolle, Confinement and entanglement dynamics on a digital quantum computer, Sci. Rep. 11, 11577 (2021).

[47] A. Schuckert and M. Knap, Probing eigenstate thermalization in quantum simulators via fluctuation-dissipation relations, Phys. Rev. Research 2, 043315 (2020).

[48] K. T. Geier and P. Hauke, From non-Hermitian linear response to dynamical correlations and fluctuation-dissipation relations in quantum many-body systems, arXiv:2104.03983 (2021).

[49] J. Vovrosh, K. E. Khosla, S. Greenaway, C. Self, M. S. Kim, and J. Knolle, Simple mitigation of global depolarizing errors in quantum simulations, Phys. Rev. E 104, 035309 (2021).

[50] R. Verdel, F. Liu, S. Whitsitt, A. V. Gorshkov, and M. Heyl, Real-time dynamics of string breaking in quantum spin chains, Phys. Rev. B 102, 014308 (2020).

[51] F. M. Surace and A. Lerose, Scattering of mesons in quantum simulators, New J. Phys. 23, 062001 (2021).

[52] P. I. Karpov, G. Y. Zhu, M. P. Heller, and M. Heyl, Spatiotemporal dynamics of particle collisions in quantum spin chains, arXiv:2011.11624 (2020).

[53] See also [108] for a QED study of meson scattering with tensor network simulations.

[54] As discussed in detail in [32], the semiclassical regime is valid deep in the ferromagnetic phase, i.e., $h<J$ in (1) and (3), when the system is not close to criticality. In the case of nearest-neighbor interactions, isolated spin domains of magnetization opposite to the longitudinal field give rise to a confining potential linear in its spatial extension. Mesonic excitations and their properties can then be estimated from the Bohr-Sommerfeld quantization condition. In a similar way, using a two-kink model, it was shown in [28] that long-range interactions give rise to an effective confining potential when restricting to the Hilbert space of two domain wall states. Details on a reformulation of the nearest-neighbor Ising model at very weak transverse field as a nonrelativistic $\mathbb{Z}_{2}$ gauge theory are discussed, e.g., in [52].

[55] A. B. Zamolodchikov, Integrals of motion and s-matrix of the (scaled) $\mathrm{T}=\mathrm{T}_{c}$ ising model with magnetic field, Int. J. Mod. Phys. A 04, 4235 (1989).

[56] R. Coldea, D. A. Tennant, E. M. Wheeler, E. Wawrzynska, D. Prabhakaran, M. Telling, K. Habicht, P. Smeibidl, and K. Kiefer, Quantum criticality in an ising chain: experimental evidence for emergent $E_{8}$ symmetry, Science 327, 177 (2010). 
[57] H. Zou et al., $E_{8}$ Spectra of Quasi-one-dimensional Antiferromagnet $\mathrm{BaCo}_{2} \mathrm{~V}_{2} \mathrm{O}_{8}$ under Transverse Field, Phys. Rev. Lett. 127, 077201 (2021).

[58] Z. Zhang, K. Amelin, X. Wang, H. Zou, J. Yang, U. Nagel, T. Room, T. Dey, A. A. Nugroho, T. Lorenz, J. Wu, and Z. Wang, Observation of E8 particles in an ising chain antiferromagnet, Phys. Rev. B 101, 220411(R) (2020).

[59] K. Amelin, J. Engelmayer, J. Viirok, U. Nagel, T. Room, T. Lorenz, and Z. Wang, Experimental observation of quantum many-body excitations of $E_{8}$ symmetry in the ising chain ferromagnet $\mathrm{CoNb}_{2} \mathrm{O}_{6}$, Phys. Rev. B 102, 104431 (2020).

[60] P. Hauke, D. Marcos, M. Dalmonte, and P. Zoller, Quantum Simulation of a Lattice Schwinger Model in a Chain of Trapped Ions, Phys. Rev. X 3, 041018 (2013).

[61] D. Yang, G. S. Giri, M. Johanning, C. Wunderlich, P. Zoller, and P. Hauke, Analog quantum simulation of (1+1)dimensional lattice QED with trapped ions, Phys. Rev. A 94, 052321 (2016).

[62] C. Muschik, M. Heyl, E. Martinez, T. Monz, P. Schindler, B. Vogell, M. Dalmonte, P. Hauke, R. Blatt, and P. Zoller, U(1) wilson lattice gauge theories in digital quantum simulators, New J. Phys. 19, 103020 (2017).

[63] Z. Davoudi, M. Hafezi, C. Monroe, G. Pagano, A. Seif, and A. Shaw, Towards analog quantum simulations of lattice gauge theories with trapped ions, Phys. Rev. Research 2, 023015 (2020).

[64] D. Paulson et al., Towards simulating 2D effects in lattice gauge theories on a quantum computer, PRX Quantum 2, 030334 (2021).

[65] Z. Davoudi, N. M. Linke, and G. Pagano, Toward simulating quantum field theories with controlled phonon-ion dynamics: A hybrid analog-digital approach, Phys. Rev. Research 3, 043072 (2021).

[66] E. Zohar, Quantum simulation of lattice gauge theories in more than one space dimension - requirements, challenges, methods, Phil. Trans. A. Math. Phys. Eng. Sci. 380, 20210069 (2021).

[67] S. Sachdev, Quantum Phase Transitions, 2nd ed. (Cambridge University Press, Cambridge, UK, 2011).

[68] T. Rakovszky, M. Mestyán, M. Collura, M. Kormos, and G. Takács, Hamiltonian truncation approach to quenches in the Ising field theory, Nucl. Phys. B911, 805 (2016).

[69] K. Hódsági, M. Kormos, and G. Takács, Quench dynamics of the Ising field theory in a magnetic field, SciPost Phys. 5, 027 (2018).

[70] Physical consequences of algebraic long-range interactions in QMB systems for eigenstate thermalization and symmetry properties are studied, e.g., in [85].

[71] D. Porras and J. I. Cirac, Effective Quantum Spin Systems with Trapped Ions, Phys. Rev. Lett. 92, 207901 (2004).

[72] N. Trautmann and P. Hauke, Trapped-ion quantum simulation of excitation transport: Disordered, noisy, and long-range connected quantum networks, Phys. Rev. A 97, 023606 (2018).

[73] P. Hauke and L. Tagliacozzo, Spread of Correlations in LongRange Interacting Quantum Systems, Phys. Rev. Lett. 111, 207202 (2013).

[74] J. A. Kjäll, F. Pollmann, and J. E. Moore, Bound states and $\mathrm{E}_{8}$ symmetry effects in perturbed quantum Ising chains, Phys. Rev. B 83, 020407(R) (2011).
[75] The mass gap $m_{n}$ of level $n$ is defined as the energy difference to the ground state, i.e., $m_{n}=E_{n}-E_{0}$.

[76] M. Lüscher, Volume dependence of the energy spectrum in massive quantum field theories, Commun. Math. Phys. 104, 177 (1986).

[77] See, e.g., [92] for an introduction.

[78] N. Wiener, Generalized harmonic analysis, Acta Math. 55, 117 (1930).

[79] A. Khintchine, Korrelationstheorie der stationären stochastischen Prozesse, Math. Ann. 109, 604 (1934).

[80] We refer to the Appendixes for further details on the finite size, long-range, and longitudinal field dependence of the energy and absorption spectra discussed in this section.

[81] X. Wang, H. Zou, K. Hodsagi, M. Kormos, G. Takacs, and J. $\mathrm{Wu}$, Spin dynamics of a perturbed quantum critical ising chain, Phys. Rev. B 103, 235117 (2021).

[82] S.-J. Gu, H.-M. Kwok, W.-Q. Ning, and H.-Q. Lin, Fidelity susceptibility, scaling, and universality in quantum critical phenomena, Phys. Rev. B 77, 245109 (2008).

[83] S.-J. Gu, Fidelity approach to quantum phase transitions, Int. J. Mod. Phys. B 24, 4371 (2010).

[84] J. Khalouf-Rivera, M. Carvajal, and F. Perez-Bernal, Quantum fidelity susceptibility in excited state quantum phase transitions: application to the bending spectra of nonrigid molecules, SciPost Phys. 12, 002 (2022).

[85] A. Russomanno, M. Fava, and M. Heyl, Quantum chaos and ensemble inequivalence of quantum long-range Ising chains, Phys. Rev. B 104, 094309 (2021).

[86] L. Cevolani, G. Carleo, and L. Sanchez-Palencia, Protected quasilocality in quantum systems with long-range interactions, Phys. Rev. A 92, 041603(R) (2015).

[87] Further details on the underlying spectra are discussed in Appendix C.

[88] A. Sørensen and K. Mølmer, Entanglement and quantum computation with ions in thermal motion, Phys. Rev. A 62, 022311 (2000).

[89] C.-C. J. Wang and J. K. Freericks, Intrinsic phonon effects on analog quantum simulators with ultracold trapped ions, Phys. Rev. A 86, 032329 (2012).

[90] P. Hauke, L. Bonnes, M. Heyl, and W. Lechner, Probing entanglement in adiabatic quantum optimization with trapped ions, Front. Phys. 3, 21 (2015).

[91] P. Schindler, D. Nigg, T. Monz, J. T. Barreiro, E. Martinez, S. X. Wang, S. Quint, M. F. Brandl, V. Nebendahl, C. F. Roos et al., A quantum information processor with trapped ions, New J. Phys. 15, 123012 (2013).

[92] J. Jensen and A. Mackintosh, Rare Earth Magnetism: Structures and Excitations, International Series of Monographs on Physics (Clarendon, Oxford, 1991).

[93] P. Nevado and D. Porras, Hidden frustrated interactions and quantum annealing in trapped-ion spin-phonon chains, Phys. Rev. A 93, 013625 (2016).

[94] S. Korenblit, D. Kafri, W. C. Campbell, R. Islam, E. E. Edwards, Z.-X. Gong, G.-D. Lin, L.-M. Duan, J. Kim, K. Kim, and C. Monroe, Quantum simulation of spin models on an arbitrary lattice with trapped ions, New J. Phys. 14, 095024 (2012).

[95] P. Nevado, S. Fernandez-Lorenzo, and D. Porras, Topological Edge States in Periodically Driven Trapped-Ion Chains, Phys. Rev. Lett. 119, 210401 (2017). 
[96] T. Manovitz, Y. Shapira, N. Akerman, A. Stern, and R. Ozeri, Quantum simulations with complex geometries and synthetic gauge fields in a trapped ion chain, PRX Quantum 1, 020303 (2020).

[97] A. Bermudez, J. Almeida, K. Ott, H. Kaufmann, S. Ulm, U. Poschinger, F. Schmidt-Kaler, A. Retzker, and M. B. Plenio, Quantum magnetism of spin-ladder compounds with trapped-ion crystals, New J. Phys. 14, 093042 (2012).

[98] S. Zippilli, M. Johanning, S. M. Giampaolo, C. Wunderlich, and F. Illuminati, Adiabatic quantum simulation with a segmented ion trap: Application to long-distance entanglement in quantum spin systems, Phys. Rev. A 89, 042308 (2014).

[99] M. Gutiérrez, M. Müller, and A. Bermúdez, Transversality and lattice surgery: Exploring realistic routes toward coupled logical qubits with trapped-ion quantum processors, Phys. Rev. A 99, 022330 (2019).

[100] B. Horstmann, B. Reznik, S. Fagnocchi, and J. I. Cirac, Hawking Radiation from an Acoustic Black Hole on an Ion Ring, Phys. Rev. Lett. 104, 250403 (2010).

[101] B. Horstmann, R. Schützhold, B. Reznik, S. Fagnocchi, and J. Cirac, Hawking radiation on an ion ring in the quantum regime, New J. Phys. 13, 045008 (2011).
[102] H.-K. Li, E. Urban, C. Noel, A. Chuang, Y. Xia, A. Ransford, B. Hemmerling, Y. Wang, T. Li, H. Häffner et al., Realization of Translational Symmetry in Trapped Cold Ion Rings, Phys. Rev. Lett. 118, 053001 (2017).

[103] M. C. Bañuls et al., Simulating lattice gauge theories within quantum technologies, Eur. Phys. J. D 74, 165 (2020).

[104] D. Zhu, S. Johri, N. M. Linke, K. A. Landsman, N. H. Nguyen, C. H. Alderete, A. Y. Matsuura, T. H. Hsieh, and C. Monroe, Generation of thermofield double states and critical ground states with a quantum computer, Proc. Natl. Acad. Sci. USA 117, 25402 (2020).

[105] J. Mildenberger, Trapped-Ion Quantum Simulations of Spin Systems at Non-Vanishing Temperature, Master thesis, Universität Heidelberg, 2019, https://www.kip.uni-heidelberg.de/ Veroeffentlichungen/details.php?id=3997.

[106] A. Rothkopf, Heavy quarkonium in extreme conditions, Phys. Rep. 858, 1 (2020).

[107] See [109] for a related study of this phenomenon from a tensor network perspective in quantum spin chains.

[108] M. Rigobello, S. Notarnicola, G. Magnifico, and S. Montangero, Entanglement generation in QED scattering processes, Phys. Rev. D 104, 114501 (2021).

[109] M. C. Bañuls, M. P. Heller, K. Jansen, J. Knaute, and V. Svensson, to appear (2022). 